\title{
Chemical Analysis: An Indispensable Means for Uncovering Severe Cases of Fraud with Cosmetics and Tattoo Inks
}

\author{
Christopher Hohl and Urs Hauri
}

\begin{abstract}
Three cases of fraud with commodities containing illegal stealth compounds are presented, which were uncovered by the State Laboratory Basel-City, Switzerland. All three commodities, grapefruit seed extracts, a phytocosmetical skin cream, and tattoo inks, were produced abroad, had forged declarations of ingredients and, in the case of the extracts and the cream, were marketed with far-reaching health claims. While inspections will identify suspicious products and would be able to eliminate health claims to some extent, only chemical analysis can uncover the illegal agents used and give law enforcement bodies the necessary evidence to immediately clamp down on those brands, where the stealth agent presents a serious health hazard to consumers.
\end{abstract}

Keywords: Analysis · Fraud · Cosmetics · Tattoo inks

\section{Introduction}

When thinking of fraud in conjunction with cosmetics, what would come to your mind: advertisements promising a skin with fewer wrinkles or face rejuvenation? Overstatement would be the more appropriate expression for these examples, especially in comparison to what law enforcement authorities have encountered while surveying the market. Surveillance is basically performed either by inspections as a means to check manufacturing or business processes, or by chemical analysis for screening the contents of products, or by using a combination of both inspection and analysis, which often proves to be most effective. In this paper we concentrate on three cases of fraud uncovered predominantly by laboratory work which otherwise would have remained undetected or at least partly unsolved.

\section{Case 1 - Benzethonium Chloride in Grapefruit Seed Extracts}

\subsection{Initial Situation}

In 1997, we encountered severe cases of fraud with so-called 'grapefruit seed extracts' (GSE). GSE are colorless liquids sold in drop dispensers, which in 1997

\footnotetext{
${ }^{\star}$ Correspondence: Dr. C. Hohl State Laboratory of Basel-City Kannenfeldstrasse 2, $\mathrm{CH}-4056$ Base E-mail: Christopher.hohl@bs.ch
}

came mainly from the USA. They were promoted with far-reaching health claims (effective in treating mycotic or bacterial infections) ascribing their efficacy to a non-toxic, broad spectrum antimicrobial substance, derived from grapefruit and therefore purely of natural origin. GSE drops were to be administered orally or on the skin. In order to define their legal status, GSE need to be categorized: Classifying them as a cosmetic is one possibility, which considers their application on skin and their usage as a mouth wash. Oral ingestion, however, would define them as a dietary supplement, which was often written on the labels. In Switzerland, these two categories are both covered by The Swiss Law on Food and Commodities. ${ }^{[1]}$ Promoted health claims on the other hand classified GSE as drugs, which are subjected to the Swiss Law on Drugs and Medical Devices. ${ }^{[2]}$ As a cosmetic or a dietary supplement, health claims made for GSE would render them illegal. As drugs, market introduction would depend on the outcome of expensive and lengthy studies and the approval by authorities, which never was the case for any GSE. Therefore, regardless of the category assigned, be it a cosmetic, a dietary supplement or a drug, the presentation of GSE as a miracle cure alone would prompt authorities to take them from the market at once. So, why bother screening GSE samples for antimicrobial substances? The answer being: because clever salesmen sometimes find a way to get around legal restrictions, as was the case for GSE: Promotion of GSE using health claims was done via printed media and in the internet, presenting the pharmacological effects of GSE in a general way without reference to a specific brand.

\subsection{Determination of Quaternary Ammonium Compounds (Quats)}

Our chemical analysis of GSE samples was first triggered by the consumer safety authority of the State Baden-Württemberg (Germany), who became aware of GSE and suspected a quaternary ammonium compound to be the active ingredient. As we had published a method shortly before, ${ }^{[3]}$ they asked us to look into the matter.

This method consists of a multi-step procedure using colorimetry with sodium bromophenol blue for sample screening, then, in the case of positive samples, a separation of quats with HPTLC using two sorbents of different selectivity (RP2 and Si 60). Identification and quantitation was done using UV-light or a color reaction with bromophenol blue (details are given in ref. [3]). Further confirmation of UVactive quats involved HPLC with a diode array UV detector on a routine basis.

In an initial survey, ten GSE samples taken from the Swiss market were checked showing that in nine of them the agent responsible for the antimicrobial activity was identified to be benzethonium chloride (BC; Fig. 1), a quaternary ammonium compound and powerful synthetic disinfectant. Levels of BC in the nine samples were between $3-11 \%$. In addition to the routine procedure, identification of benzethonium was verified with LC/MS and H-NMR.

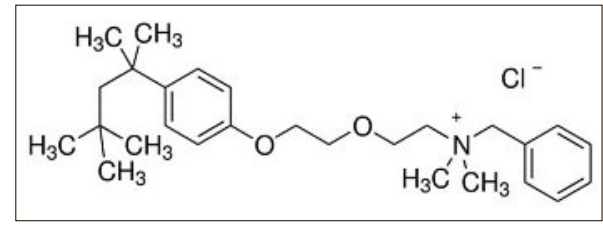

Fig. 1. Benzethonium chloride 


\subsection{Benzethonium-containing Samples - Assessment and Implications}

From a consumer safety standpoint, GHS (Globally Harmonized System of Classification and Labelling of Chemicals) Hazard Statements show BC to be toxic if swallowed and caustic on contact resulting in severe skin and eye burns. ${ }^{[4]}$ In Europe, the current limit for its usage in cosmetics is $0.1 \%$, except for oral care products, where $\mathrm{BC}$ is not tolerated at all. Its usage in dietary supplements is generally forbidden. ${ }^{[5]}$ Whatever category BCcontaining GSE are allocated to, be it a cosmetic, a dietary supplement or a drug, their presence on the market was clearly illegal. With positive samples exceeding the limit for cosmetics by a factor of 30 up to 110 , a health hazard for consumers had to be considered as imminent. Measures taken by competent authorities across Switzerland were therefore an immediate ban of objected products. In addition, the Swiss Federal Office of Public Health informed consumer organizations and business branches potentially involved in the marketing of GSE among others. ${ }^{[6]}$ Media reported prominently on this topic.

\subsection{Aftermath}

In the following two years, GSE products were still sporadically found on the Swiss market and sent to us for testing. Besides liquid samples, we then also encountered chewing tablets. While no antimicrobial agents were found in the tablets we tested, liquids sometimes contained benzalkonium chloride (BAC; Fig. 2), a mixture of synthetic alkylbenzyl dimethyl ammonium cations with even-numbered alkyl chain lengths between $\mathrm{C} 8$ and $\mathrm{C} 18$, in levels comparable to those found for benzethonium chloride (4-11\%). Being structurally similar to BC, BAC is also a powerful synthetic disinfectant, toxic if swallowed and caustic on contact resulting in severe skin and eye burns.[7] Usage in cosmetics as a preservative is limited to $0.1 \%$. Rinseoff haircare products may contain up to $3 \%$. Measures for BAC-containing GSE products were therefore the same.

Following public awareness, a medical doctor from an ophthalmic clinic informed us of three incidents where a mix-up of eye drops with GSE led to eye injuries. This information gave a rare link between measures taken by consumer safety authorities

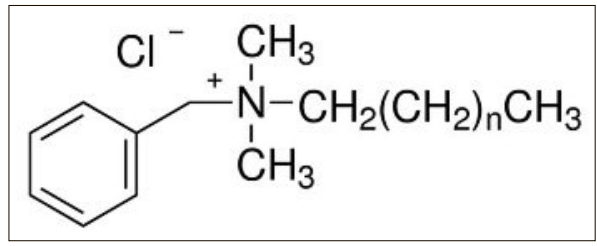

Fig. 2. Benzalkonium chloride and a resulting positive effect on health costs and prevention of suffering.

\section{Case 2: Triamcinolone Acetonide in a Phytocosmetic Skin Cream}

\subsection{Initial Situation}

Patients with skin disorders often prefer ointments containing ingredients of herbal origin to those having a synthetic corticosteroid as the active agent. While prolonged or inappropriate use of topical corticosteroids may provoke adverse reactions such as skin thinning, natural ointments are arbitrarily thought to be free of any side effects. Repeatedly, however, natural ointments were found to contain stealth corticosteroids. In 2000, Professor Andreas Bircher, a medical doctor from the University Hospital of Basel requested our support in solving the following case (for details refer to ref. [8]): Patients told him how they successfully treated dermatitis using a phytocosmetic skin cream which was not officially on the market in Switzerland. They purchased the cream either abroad or in pharmacies, which imported it on behalf of the patients. Labelling did not mention any agent known to be effective in treating skin disorders. Professor Bircher then tested the cream in a clinical study on more than 20 patients showing it to be highly effective and therefore suspected the cream to contain a corticosteroid. He asked us to investigate and provided us with cream samples of different lots.

\subsection{Determination of Corticosteroids in 'Cosmetics'}

Samples are first dissolved or suspended in ethanol and also in acetone. If necessary, $N, N$-dimethylformamide or a mixture of ethanol/water are used to better dissolve or suspend samples. Analysis is then performed with LC/DAD/MS. The method is capable of screening for 40 corticosteroids down to the lower $\mu \mathrm{g} / \mathrm{g}$ range (for details refer to ref. [9]).

The screening of the cream samples revealed low amounts of triamcinolone acetonide (TCA; Fig. 3) in all lots. Levels ranged from 16 to $40 \mu \mathrm{g} / \mathrm{g} .{ }^{[8]}$

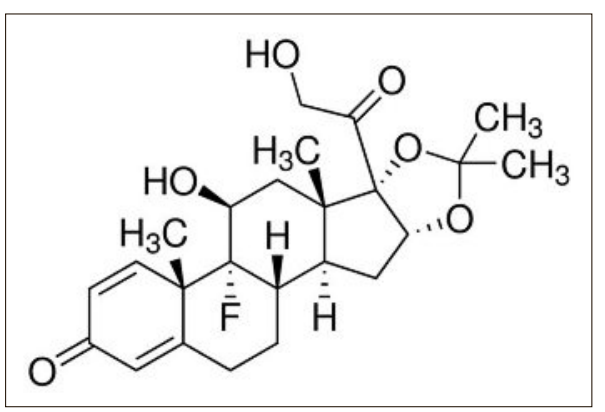

Fig. 3. Triamcinolone acetonide

\subsection{Triamcinolone Acetonide- containing Samples - Assessment and Implications}

TCA is a potent synthetic corticosteroid used in prescription drugs for treating various skin conditions. Medical creams typically contain $100-1000 \mu \mathrm{g} / \mathrm{g}$. Levels found in the phytocosmetic cream were therefore somewhere between two and six times lower than low-dosed medical creams. The study on patients showed that these levels were still effective. According to regulations, the presence of corticosteroids in cosmetics is illegal.[10] What was the cause for the presence of TCA? Contamination as a cause was ruled out as TCA was found in all sampled lots in the low ppm range. Checking the internet further revealed that the cream was (illegally) marketed as a natural remedy for treating skin disorders. The obvious conclusion was that TCA was intentionally added as a stealth active ingredient. Being sold on an uncontrolled market with wrongful assertions to consumers, unaware of potential adverse effects, prompted health authorities to immediately interdict any import.

\subsection{Aftermath}

In the wake of our investigation, the European general distributor of the cream took legal action against the producer. In 2005 we were asked to analyze new samples of the same product which were claimed to be free of TCA. This time, no corticosteroids were found. In a subsequent clinical study, the plain version of the cream proved to be ineffective for treating skin disorders while the same cream spiked with $25 \mu \mathrm{g} / \mathrm{g}$ triamcinolone acetonide did show the expected effects. ${ }^{[11]}$

\section{Case 3: Illegal Pigments in Tattoo Inks}

\subsection{Initial Situation}

Pigments are colorants which are more or less insoluble in common organic solvents and water. Their bioavailability is often assumed to be negligible and toxicity therefore of no concern. This assumption, however, does not hold in general. In the case of tattoo inks, pigments are injected into the skin and are intended to stay there lifelong. Adverse reactions of the human body to some of these pigments, e.g. allergies, dermatitis and many others are fairly common and well documented. Regulations on tattoo inks therefore designate many colorants as illegal and stipulate a full declaration of constituents on the labels. ${ }^{[12]}$ Our laboratory is focused on the determination of organic compounds in tattoo inks and 2009, 2012, and 2014 saw us taking the lead in federal surveillance activities on inks found on the Swiss market. ${ }^{[13-15]}$ 


\subsection{Determination of Pigments in Tattoo Inks}

Our procedure (publication pending) mainly relies on LDI-TOF-MS for pigment identification. After shaking, an aliquot of ink sample is analyzed without matrix addition with LDI-TOF-MS. Measurements are supplemented and confirmed by UV/ Vis colorimetry using sulfuric acid, chloronaphthalene or methylnaphthalene as a solvent as well as by HPLC/DAD/ MS after dissolving pigments in $N, N$ dimethylformamide or other appropriate solvents. As a result of the federal actions, $20 \%$ or more of all tattoo inks tested had to be banned from sale due to illegal organic pigments. Some of these inks had labels declaring legal pigments but actually contained illegal pigments instead. Here are just a few examples:

- Several green inks with declared C.I. 74265 (also known as Pigment Green 36) contained C.I. 74260 (Pigment Green 7) instead. The same illegal pigment was also found in inks where mixtures of blue and yellow colorants were declared.

- Several magenta and purple inks with combinations of blue and a red pigments labelled on the package contained illegal magenta (C.I. 73900 (Pigment Violet 19), C.I. 73915 (Pigment Red 122)) or purple pigments (C.I. 51319 (Pigment Violet 23)).

While an experienced inspector will identify those samples as suspicious where the color tone of the sample does not match with the labelled colorants, only chemical analysis can detect more sophisticated cases where labelled colorants and color shade are consistent.

\subsection{Wrongful Labelling - Assessment and Implications}

To put things into the right perspective: none of the pigments used in tattoo inks - neither legal or illegal - have ever been tested to ensure their health safety when injected into the skin. This also applies to all the other constituents, because none of them have ever been intentionally produced for their usage in tattoo inks. Current regulations throughout Europe, however, tolerate the fact that no ink can be regarded as safe for consumers. Nevertheless, forging tattoo ink labels in an attempt to cover up illegal pigments and deceive clients and authorities must be seen as selling a hazardous product to unsuspecting consumers. The dimension of this fraud must not be underestimated as some of the companies involved are among the major producers of tattoo inks. As label forging was done abroad with Swiss importers being deceived as well, it was unreasonable to hold them responsible for the moment. The future, however, will see them improve their self-monitoring of imported inks.

\section{Final Conclusions}

In all three cases, the use of clandestine compounds went together with a fraudulent declaration. In the case of GSE and the skin cream, where compounds were added for a pharmacological effect, marketing made use of illegal health claims and presented the product to be entirely of natural origin. In the case of tattoo inks, the motive was probably to stick to the original product composition that was developed before the regulation came into force. What was the driving force behind these frauds? A reasonable assumption would be ruthless profit maximization by eluding legal restrictions on compounds and avoiding elaborate safety assessments as well as lengthy approval procedures. The presented cases also show that the use of stealth compounds can result in a serious health hazard for consumers.

Was chemical analysis really indispensable in uncovering the presented frauds? Yes indeed, we think so, because in all cases, the responsible producer was abroad with production facilities and documentation therefore being out of reach for inspection. These are the moments when chemical analysis really comes to bear in full force!

Received: January 18, 2016

[1] Schweizer Lebensmittel- und Gebrauchsgegenständeverordnung (LGV; SR 817.02) vom 23. November 2005, Stand vom 1.1.2016
[2] Schweizer Bundesgesetz über Arzneimittel und Medizinprodukte (Heilmittelgesetz, HMG; SR 821.21) vom 15.12.2000, Stand vom 1.1.2014

[3] U. Schlegel, C. Hohl, C. Bürgi, Mitt. Lebensm. Hyg. 1997, 88, 191.

[4] Benzthonium chloride, European Chemicals Agency (ECHA), Information on Chemicals, Classification and Labelling, http://echa. europa.eu/home, accessed Jan. 18, 2016.

[5] Schweizer Verordnung des EDI über die in Lebensmitteln zulässigen Zusatzstoffe (Zusatzstoffverordnung, ZuV, SR 817.022.31) vom 23.11.2005, Stand vom 1.10.2015.

[6] Bundesamt für Gesundheit, 'Benzethonium in Grapefruitkern-Extrakten', Informationsschreiben Nr. 15/1997, http://www.blv.admin. ch/themen/04678/04802/04923/index.html?lan $g=$ de $\&$ download $=\mathrm{NHz}$ LpZig7t, lnp6IONTU042 l2Z6ln1acy4Zn4Z2qZpnO2Yuq2Z6gpJCGdHx4 fGym162dpYbUzd,Gpd6emK2Oz9aGodetmqa N19XI2IdvoaCUZ,s-, accessed Jan. 18, 2016.

[7] Benzyl-C12-14-alkyldimethylammonium chlorides, European Chemicals Agency (ECHA), Information on Chemicals, Classification and Labelling, http://echa. europa.eu/home, accessed Jan. 18, 2016.

[8] A. J. Bircher, U. Hauri, M. Niederer, C. Hohl, C. Surber, Br. J. Dermatol. 2002, 146, 524.

[9] U. Hauri, C. Hohl, Mitt. Lebensm. Hyg. 2004, 95, 466.

[10] Schweizer Verordnung des EDI über Kosmetische Mittel (VKos; SR 817.023.31) vom 23. November 2005, Stand vom 1.10.2015, Annex 2, Number 300.

[11] C. Pellanda, M. Weber, A. Bircher, C. Surber, Dermatology 2005, 211, 338.

[12] Schweizer Verordnung des EDI über Gegenstände für den Schleimhaut-, Haut- und Haarkontakt sowie über Kerzen, Streichhölzer, Feuerzeuge und Scherzartikel (Verordnung über Gegenstände für den Humankontakt, HKV, 817.023.41) vom 23. November 2005, Stand vom 1.7.2015, Article 8.

[13] K. W. Lüthi, J. Amberg, A. Baumgartner, B Brüschweiler, S. Gautsch, U. Hauri, BAG Bull. 2009, 29, 535.

[14] U. Hauri, Joint campaign by the Swiss Association of Cantonal Chemists (VKCS) with financial support from the Swiss Federal Office of Public Health, 'Inks for tattoos and PMU (permanent make-up) / Organic pigments, preservatives and impurities such as primary aromatic amines and nitrosamines', http:// www.kantonslabor.bs.ch/dms/kantonslabor/ download/berichte/berichte-2011/JB_Tattoo_ PMU 2011_EN.pdf, accessed April 14, 2016.

[15] U. Hauri, Tinten für Tattoo und PMU - National investigation campaign, 'Inks for tattoos and permanent make-up', initiated by the Swiss Association of Cantonal Chemists, Kantonales Laboratorium Basel-Stadt 2014, http:// www.kantonslabor.bs.ch/dms/kantonslabor/ download/berichte/berichte-2014/Tattoo_ PMU_2014_EN-UK-/Tattoo_PMU_2014_ EN\%28UK\%29.pdf, accessed April 14, 2016. 